\title{
Electrically pumped two-dimensional Bragg grating lasers
}

\author{
Lin Zhu, John M. Choi, Guy A. DeRose, Amnon Yariv, and Axel Scherer \\ Department of Electrical Engineering, California Institute of Technology, 1200 East California Boulevard, \\ Pasadena, California 91125
}

\begin{abstract}
Received February 1, 2006; revised April 4, 2006; accepted April 5, 2006; posted April 7, 2006 (Doc. ID 67592) We demonstrate electrically pumped InGaAsP two-dimensional Bragg grating (2DBG) lasers with two line defects. The $2 \mathrm{DBG}$ structure uses a weak $2 \mathrm{D}$ index perturbation surface grating to control the optical modes in the plane of the wafer. Measurements of the 2DBG lasers show that modal control in both the longitudinal and transverse directions is due to the gratings and defects. The 2DBG lasers are promising candidates for single-mode, high power, and high efficiency large-area lasers. (C) 2006 Optical Society of America OCIS codes: $350.2770,130.2790,140.5960$.
\end{abstract}

It is well known that a grating along the length of a laser can control its longitudinal modes, as in a distributed feedback (DFB) laser. ${ }^{1}$ Recently, we have demonstrated theoretically and experimentally that a transverse Bragg grating can control the optical modes across the width of the laser for efficient, stable, single lateral mode operation even at high powers. $^{2-4}$ In Ref. 4, electrically pumped transverse Bragg resonance (TBR) lasers showed a reduced threshold current density compared with gain-guided broad area lasers, suggesting that significant efficiency improvements may be possible. However, a TBR laser has multiple longitudinal modes because the longitudinal feedback mechanism is provided by the Fabry-Perot resonances from the reflection at the end facets.

In this Letter, we demonstrate a two-dimensional Bragg grating (2DBG) structure with two quarterwave slip line defects to control the optical modes in both longitudinal and transverse directions by incorporating a longitudinal Bragg grating into a TBR waveguide. The resulting $2 \mathrm{DBG}$ laser makes single transverse and longitudinal mode operation possible through the proper design of the gratings and defects. Unlike conventional 2D photonic crystal lasers, ${ }^{5,6}$ which use a large refractive index perturbation to confine light in a plane, the 2DBG structures described here selectively control longitudinal and transverse wave vector components using a weak index perturbation. Thus the optical modes confined by the grating in a 2DBG laser will spread out into the periodic active medium, allowing for high power operation.

Figure 1 shows a schematic of a typical structure of a 2DBG laser. The laser consists of a rectangular lattice array of air holes with two line defects in a thin slab that includes active multiple-quantum-well layers. In the limit of weak index perturbation, which obtains, for example, for sufficiently small hole diameter, the optical mode for the proposed structure can be separated into transverse $(x)$, vertical $(y)$, and longitudinal $(z)$ components. In the wafer plane $(x-z)$, a mode that satisfies both transverse and longitudinal Bragg resonance conditions will be confined because of the distributed Bragg reflection. Light that does not satisfy the Bragg conditions will be lost. This
Bragg condition can be expressed as $k_{x}=l(\pi / a), k_{z}$ $=j(\pi / b) \quad(l \neq 0, j \neq 0)$, where $k_{x}$ is the transverse wave vector, $k_{z}$ is the longitudinal wave vector, $a$ is the transverse grating period, $b$ is the longitudinal grating period, and $l$ and $j$ are the orders of the grating. Because the vertical wave vector $k_{y}$ is determined by the wafer epitaxial layer structure, $k_{x}$ and $k_{z}$ satisfy $k_{x}^{2}+k_{z}^{2}=n_{\text {eff }}^{2} k_{0}^{2}$, where $n_{\text {eff }}$ is the effective refractive index for the optical mode of the wafer structure. In our design, we chose $k_{z} \approx n_{\text {eff }} k_{0}$. Two line defects perpendicular to each other are introduced in the $2 \mathrm{DBG}$ to define the optical resonance condition in the longitudinal and transverse directions. Thus the widths of two line defects should satisfy ${ }^{1,2} W_{1}$ $=(2 m+1) a / 2 l$ and $W_{2}=(2 n+1) b / 2 j$, where $W_{1}$ is the transverse defect width, $W_{2}$ is the longitudinal defect width, and $m$ and $n$ are integers (see Fig. 1).

For practical 2DBG laser design, we used a firstorder grating for the transverse direction with a period of $1 \mu \mathrm{m}$ and a second-order grating for the longitudinal direction with a period of $480 \mathrm{~nm} . W_{1}$ and $W_{2}$ were chosen to be $0.5 \mu \mathrm{m}$ and $120 \mathrm{~nm}$, respectively. Since the index contrast of the gratings in both longitudinal and transverse directions was weak, a single laser device had a relatively large area of $1.0 \mathrm{~mm} \times 0.16 \mathrm{~mm}$ consisting of about 320,000 small holes, each with a radius of $100 \mathrm{~nm}$. The large differ-

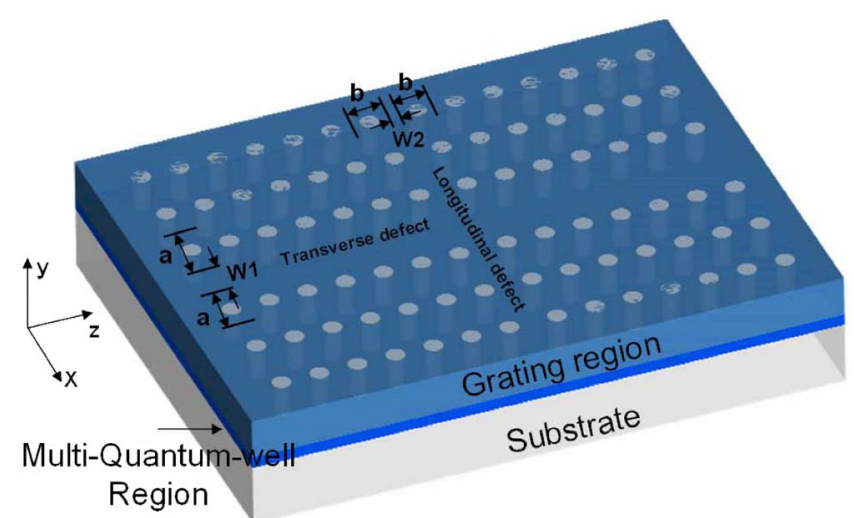

Fig. 1. (Color online) An illustration of a 2DBG laser with two line defects. $a$ is the transverse grating period, $b$ is the longitudinal grating period, $W_{1}$ is the transverse defect width, and $W_{2}$ is the longitudinal defect width. 


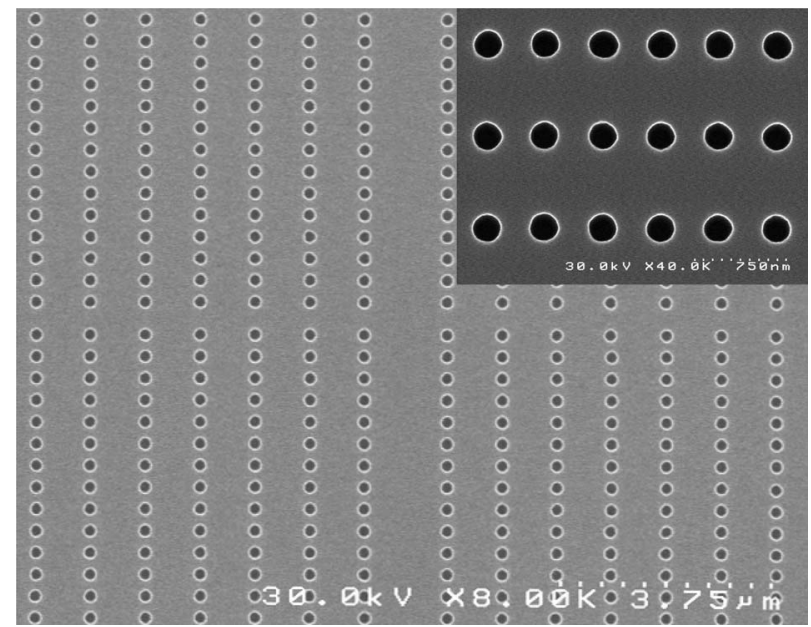

Fig. 2. SEM image of a 2DBG laser. Inset, details of the air holes, each with a radius of $100 \mathrm{~nm}$. The image was taken after a RIE step through the InGaAsP wafer before the deposition of electrical contacts.

ence in scale between the device area and the hole area, nearly seven orders of magnitude, presented challenges for the optimization of the fabrication process.

We fabricated 2DBG lasers in commercially grown, active semiconductor materials. The semiconductor material had InGaAsP layers totaling a thickness of $725 \mathrm{~nm}$ on top of an InP substrate. The InGaAsP layers included four $75 \AA$ wide unstrained InGaAsP quantum wells with peak photoluminescence occurring at $1548 \mathrm{~nm}$. The quantum wells are located at $675 \mathrm{~nm}$ from the top wafer surface. The grating was formed on the surface of the wafer without etching through the multiple-quantum-well region, so that the coupling constant was fairly small in each direction. In the coupled-mode calculations, we defined weak coupling to be when $\kappa<0.1 / \mu \mathrm{m}$, which is satisfied when the bottom of the etched holes is more than $150 \mathrm{~nm}$ away from the quantum well region in our wafer structure.

The desired 2DBG structures were defined by electron-beam lithography in a $120 \mathrm{~nm}$ thick $\mathrm{SiO}_{2}$ layer deposited by plasma-enhanced chemical vapor deposition (PECVD) on the top of the wafer. We exposed polymethylmethacrylate (PMMA) resist in a Leica Microsystems EBPG 5000+ electron beam writer at $100 \mathrm{kV}$. A proximity correction code was used for the electron beam lithography to obtain a uniform pattern distribution over a large area. After development, the PMMA patterns were transferred into the $\mathrm{SiO}_{2}$ layer by reactive ion etching (RIE) using $\mathrm{CHF}_{3}$ plasma. The $\mathrm{SiO}_{2}$ layer then served as a hard mask to etch the semiconductor surface grating using an inductively coupled plasma RIE with HI/Ar chemistry. ${ }^{7}$ The remaining $\mathrm{SiO}_{2}$ was then stripped off in a buffered hydrofluoric acid solution. The $p$-type electrical contacts, AuZn/Au, were thermally evaporated on the device. After mechanical lapping, the $n$-type contacts, $\mathrm{AuGe} / \mathrm{Au}$, were applied. Figure 2 shows a scanning electron microscope (SEM) image of a $2 \mathrm{DBG}$ device.
The semiconductor etch depth determines the grating strength, and is therefore a critical parameter for the device performance. It also affects the optical loss, since the metal contact layer was directly deposited on the wafer surface. A deep etch depth leads to a strong grating strength, but increases the modal loss, because the metal in the etched holes gets close to the quantum well layer underneath. For our devices, a surface grating depth of $430 \mathrm{~nm}$ was found to provide optimal performance. The theoretical coupling constants from coupled mode theory are about $0.04 \mu \mathrm{m}$ in the transverse direction and $0.003 \mu \mathrm{m}$ in the longitudinal direction.

The laser bars were cleaved to lengths of about $1 \mathrm{~mm}$ and tested in pulsed operation with no active cooling. Current pulses with a duration of $50 \mathrm{~ns}$ and a period of $50 \mu \mathrm{s}$ were injected to drive the lasers. The light-current density curve is shown in Fig. 3 with a clear threshold occurring at $442 \mathrm{~A} / \mathrm{cm}^{2}$, indicating the onset of laser action. Laser spectra above the threshold obtained under several pump currents are illustrated in Figs. 4(a)-4(c), showing multiple laser modes. The spectrum of a TBR laser is shown in Fig. 4(d) for comparison.

Figure 3(a) shows the optical spectrum just above the laser threshold. The free spectral range (FSR) is about $0.36 \mathrm{~nm}$, corresponding to a length of $1.04 \mathrm{~mm}\left[L=\lambda^{2} / 2 n_{g} \lambda_{\mathrm{FSR}}\right.$ (Ref. 8); $n_{g}$ is assumed to be 3.2]. The FSR suggests that these emission peaks arose from the longitudinal modes of the laser defined by end facets. Two main groups of laser modes are observed. One is centered around $1548.6 \mathrm{~nm}$, at the peak of the material gain spectrum, and the other is centered around $1552.1 \mathrm{~nm}$, determined by the grating structure. The wavelength of the main lasing peak remains at $1552.1 \mathrm{~nm}$ under several pump currents, in contrast to the TBR lasers in Ref. 4. Laser modes around the gain spectrum peak were suppressed under higher pump currents, as shown in Fig. 4(b) at a pumping current density of $J=1.2 \mathrm{Jth}$. The spectrum shows that the modes selected by the

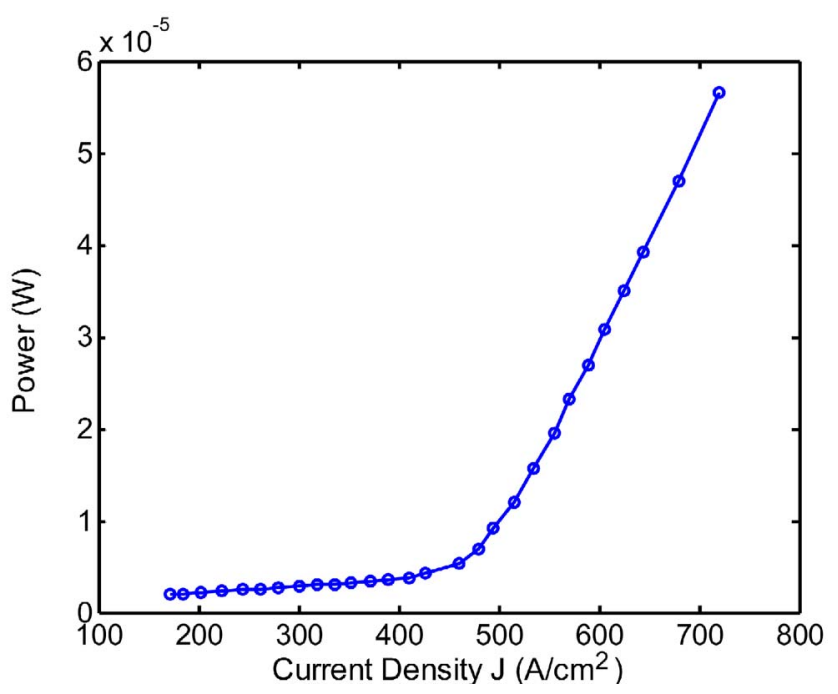

Fig. 3. (Color online) Light-current density curve with a clear threshold occurring at $442 \mathrm{~A} / \mathrm{cm}^{2}$ (average power versus peak current density). 

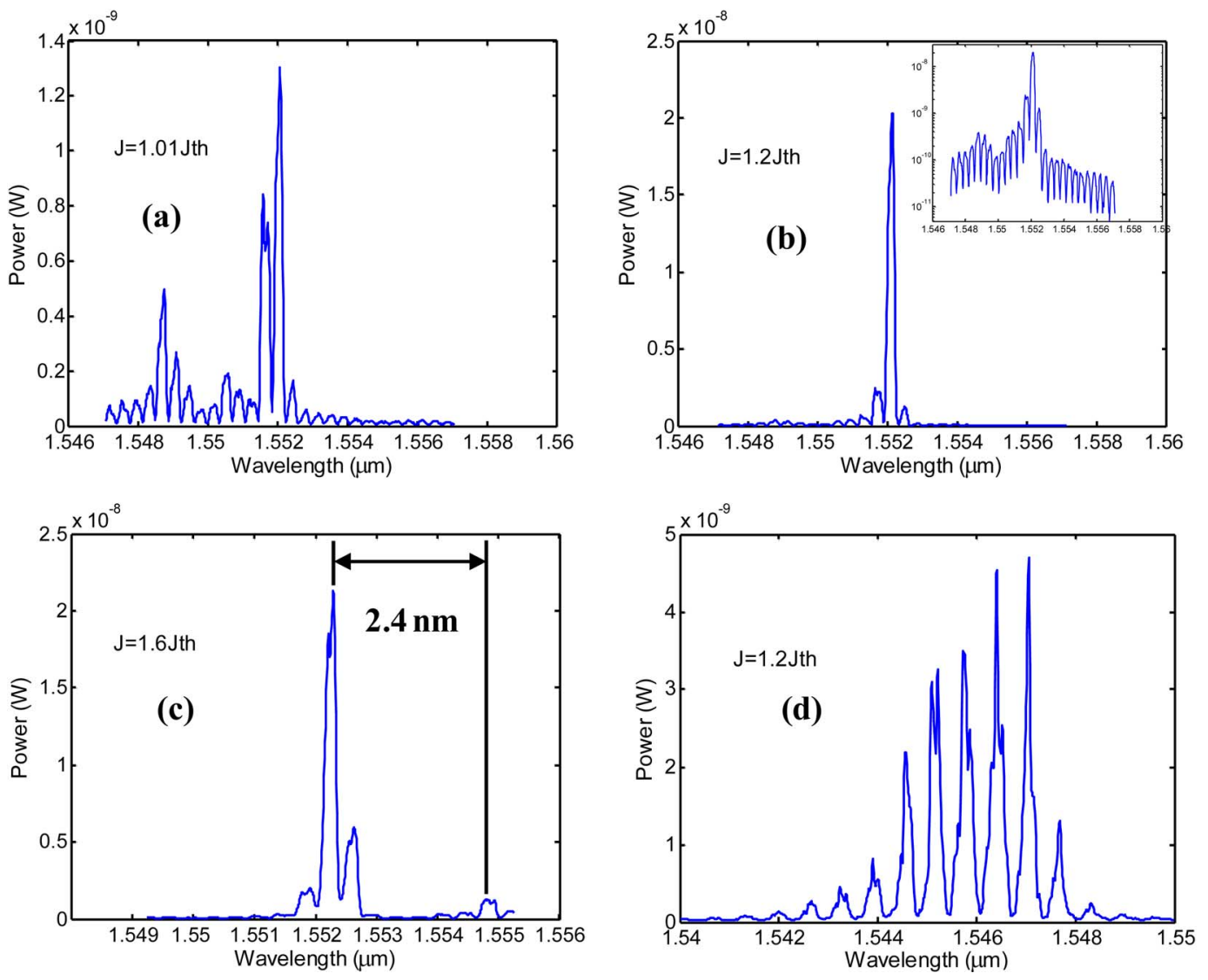

Fig. 4. (Color online) (a)-(c) Laser spectra above the threshold obtained under several pump currents. (d) Spectrum of a TBR laser at 1.3Jth pump current. Inset of (b), spectrum in logarithmic scale.

grating dominated over other Fabry-Perot modes. Compared with a typical TBR laser spectrum in Fig. 4(d), which is similar to a broad area laser spectrum, Fig. 4(c) proves that the grating in the fabricated 2DBG laser can control the longitudinal modes. Since we did not apply antireflection coatings at the end facets, some longitudinal modes due to the FabryPerot resonances from the facet reflection still remained around the main laser peak.

Figure 4(c) shows the emission spectrum at the highest pump current we obtained. Further increase of the pump current damaged the device. The line width of the laser modes increased due to heating effects. A new mode at $1554.6 \mathrm{~nm}$ appeared. The spacing between the main peak and this new emission peak is $2.4 \mathrm{~nm}$, corresponding to a length of about $160 \mu \mathrm{m}$, which is the exact width of the $2 \mathrm{D}$ grating. The spacing indicates that this lasing is due to a new transverse mode. It also suggests that, in the transverse direction, the laser modes are confined by the 2DBG structure. The presence of multiple transverse modes can be attributed to the broad bandwidth of the transverse grating, which leads to the low modal discrimination.

In summary, we have demonstrated larger-area, edge-emitting 2DBG lasers in InGaAsP active semiconductor materials with a $2 \mathrm{D}$ grating. Lasing was achieved at room temperature under pulsed electrical pumping. Our measurements demonstrate that 2DBG lasers can effectively control the lasing modes in both longitudinal and transverse directions through the proper design of the gratings and defects, which is the first step toward obtaining a single mode device under cw operation.

L. Zhu thanks Oskar Painter and Raviv Perahia for providing access to their PECVD facility. William Green, Reginald Lee, and Joyce Poon are also acknowledged for fruitful discussions. The work was supported by the Air Force Office of Scientific Research (H. Schlossbeg) and by DARPA (M. Stickley). L. Zhu's e-mail address is linz@caltech.edu.

\section{References}

1. M. Nakamura and A. Yariv, Appl. Phys. Lett. 22, 515 (1973).

2. A. Yariv, Opt. Lett. 27, 936 (2002).

3. A. Yariv, Y. Xu, and S. Mookherjea, Opt. Lett. 28, 176 (2003).

4. J. M. Choi, L. Zhu, W. Green, G. Derose, and A. Yariv, "Large-area semiconductor transeverses Bragg resonance (TBR) lasers for efficient, high power operation," in ICALEO 2005 24th International Congress on Applications of Lasers and Electro-optics, (Laser Institute of America, 2005), paper \#406.

5. M. Loncar, T. Yoshie, A. Scherer, P. Gogna, and Y. Qiu, Appl. Phys. Lett. 81, 2680 (2002).

6. H. G. Park, S. H. Kim, S. H. Kwon, Y. G. Ju, J. K. Yang, J. H. Baek, S. B. Kim, and Y. H. Lee, Science 305, 1444 (2004).

7. W. Green, J. Scheuer, G. DeRose, and A. Yariv, Appl. Phys. Lett. 85, 3669 (2004).

8. A. Yariv and P. Yeh, Optical Waves in Crystals (Wiley Interscience, 2003). 